\title{
Synthesis of RF Circuits with Negative Time Delay by Using LNA
}

\author{
Blaise Ravelo \\ IRSEEM (Institut de Recherche en Systèmes Electroniques Embarqués), EA 4353, ESIGELEC, \\ Avenue Galilée, 76801 Saint Etienne du Rouvray, France. \\ Tel.: +33 (0)2 32915971 / +33 (0)6 45334722 \\ Fax: +33 (0)232915859 \\ *corresponding author, E-mail: blaise.ravelo@yahoo.fr
}

\begin{abstract}
A demonstration of the negative time-delay by using active circuit topologies with negative group delay (NGD) is described in this paper. This negative time delay is realized with two different topologies operating in base band and modulated frequencies. The first NGD topology is composed of an RL-network in feedback with an $\mathrm{RF} /$ microwave amplifier. Knowing the characteristics of the amplifier, a synthesis method of this circuit in function of the desired NGD values and the expected time advance is established. The feasibility of this extraordinary physical effect is illustrated with frequency- and time-domain analyses. It is shown in this paper that by considering an arbitrary waveform signal, output in advance of about $7 \mathrm{~ns}$ is observed compared to the corresponding input. It is stated that such an effect is not in contradiction with the causality. The other NGD topology is comprised of a microwave amplifier associated with an RLC-series resonant. The theoretical approach illustrating the functioning of this NGD circuit is established by considering the amplifier Sparameters. Then, synthesis relations enabling to choose the NGD device parameters according to the desired NGD and gain values are also established. To demonstrate the relevance of the theoretic concept, a microwave device exhibiting NGD function of about $-1.5 \mathrm{~ns}$ at around $1.19 \mathrm{GHz}$ was designed and analyzed. The NGD device investigated in this paper presents advantages on its faculty to exhibit positive transmission gain, the implementation of the bias network and matching in the considered NGD frequency band.
\end{abstract}

Keywords: Negative group delay (NGD), active topology, time-advance.

\section{Introduction}

Recent studies revealed that certain passive [1-8] and active [9-18] circuits are susceptible to exhibit the negative group delay (NGD) function at low- and RF-/microwavefrequencies. Theoretical and experimental analyses confirm that this innovative physical function allows to generate an output signal with wave fronts propagating in advance of its input under certain conditions [7-14]. Inversely to the ordinary medium, in this case, the time delay can be assumed as negative [4-5, 9-16]. It was pointed out [19-20] that this NGD effect does not forbid the causality principle. The limits and physical meaning of the negative time delay in function of the NGD transfer function parameters are stated in [21-22].

At the beginning, the occurrence of the NGD was initially explained with the anomalous dispersion [23-27]. The original theoretical study on the abnormal media with negative group velocity is introduced in [24]. In fact, it was shown that at certain wavelengths, these media is capable to present refractive group index $n_{g}$ can be negative. The theoretical demonstration of the NGD can be illustrated with the following simple mathematical analysis. First of all, it is well-known that the group velocity is linked to the speed of light in the vacuum $c$ by the expression:

$$
v_{g}=c / n_{g}
$$

Clearly, if $n_{g}$ is negative, the group velocity $v_{g}$ is also negative. It means that when using a physical medium with geometrical length $d$, the group delay, by definition given by:

$$
\tau_{g}=d / v_{g}
$$

can also be negative. Because of the inherent fact of the wave reshaping or the combination of the constructive and destructive interferences at the edge of the abnormal dispersive passive medium, the NGD phenomenon is systematically accompanied with significant losses, in particular, at microwave wavelengths [1-8]. So, the applications of NGD passive circuits are still limited before the middle 2000s.

To overcome this limitation, active microwave topologies based on the RF/microwave transistors have been developed [14-18, 21-22]. Then, different applications of the NGD function were proposed in the literature. One of the most published applications are the design of independent frequency NGD phase shifter [28-31] and broadband active balun [32], feed forward amplifier [33-34], microwave circuit with pulse compression [35] and also the reduction 
of the propagation delay in the microelectronic interconnect with the NGD equalization technique [36-40].

However, because of the transistor access-matching difficulties, the complexity of the biasing networks and the output voltage sign inversion compared to the input is found with certain NGD topologies as proposed in [14-18]. It means that somehow the use of the transistors is more and more sophisticated. To avoid the complexity of bias network and the issues related to the matching level, the replacement of the transistor by an integrated low noise amplifier (LNA) was introduced in [41] for the base band applications.

The present paper is focused on the study of NGD topologies using microwave LNA. For the better understanding, this paper is organized in three main sections. Section 2 presents the analysis of the base band NGD topology proposed briefly in [41]. This first NGD circuit is aimed to the base band applications. Section 3 is focused on the investigation of the NGD LNA topology dedicated to the modulated microwave signals application. Section 4 is the general conclusion of the paper.

\section{Investigation on an NGD circuit with LNA operating in baseband frequencies}

Figure 1 presents the topology of the NGD circuit understudy for the base band frequencies. It is comprised of an RL-network in feedback with an LNA $\boldsymbol{A}$.

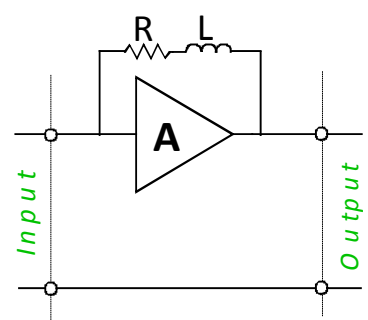

Figure 1: Amplifier in feedback with an RL-series network.

In order to achieve a global perspective on the frequency analysis including the stability, transfer function and matching access, the present analytical approach is based on the S-parameters analysis. To do this, an ideal microwave LNA operating in base band frequencies is considered. For the sake of the analytical expression simplification, in the remainder of this section, the considered amplifier is substituted by the ideal S-parameters expressed as:

$$
S_{A}=\left[\begin{array}{ll}
\rho & 0 \\
T & \rho
\end{array}\right]
$$

where $\rho$ and $T$ represent respectively the return loss and transmission parameter. Moreover, this amplifier is supposed perfectly unilateral, $S_{A 12}=0$. The theoretical analysis of this topology by using the S-parameters is introduced in the next subsection. Then, numerical demonstrations of the negative time delay will be explored based on the analyses in frequency- and time-domains.

\subsection{Theory on the baseband topology proposed}

After the calculation of the S-parameters of the cell shown in Figure 1, we will establish the synthesis relations enabling to determine the elements $R$ and $L$ in function of the desired gain and NGD values knowing the characteristics of the amplifier.

\subsubsection{S-parameter analysis of the NGD cell for base band frequencies}

As the circuit proposed is comprised of an RL-network in feedback with an amplifier, it is easier to calculate the Sparameters from the admittance matrix. According to the circuit and system theory, the admittance matrix of the whole circuit which is also the sum of the passive part admittance matrix and that of the amplifier is expressed as:

$$
[\underline{Y}]=\left[\begin{array}{ll}
\underline{Y}_{11}(j \omega) & \underline{Y}_{12}(j \omega) \\
\underline{Y}_{21}(j \omega) & \underline{Y}_{22}(j \omega)
\end{array}\right],
$$

with

$$
\begin{aligned}
\underline{Y}_{11}(j \omega) & =\underline{Y}_{22}(j \omega)=\frac{1}{R+j L \omega}-\frac{\rho-1}{R_{0}(\rho+1)}, \\
\underline{Y}_{12}(j \omega) & =\frac{-1}{R+j L \omega}, \\
\underline{Y}_{21}(j \omega) & =\frac{-1}{R+j L \omega}-\frac{2 T}{R_{0}(\rho+1)^{2}},
\end{aligned}
$$

where $\omega$ is the angular frequency, $j=\sqrt{-1}$ and $R_{0}=50 \Omega$ is the reference impedance of the source and the load. Through the admittance to S-matrix transform, one establishes that the following S-parameters of the NGD cell understudy are:

$$
\begin{gathered}
\underline{S}_{11}(j \omega)=\underline{S}_{22}(j \omega)=\frac{R_{0}(T-1)+\rho\left(2 \underline{Z}+\rho \cdot R_{0}\right)}{2 \underline{Z}+R_{0}(2 \rho-T+2)}, \\
\underline{S}_{12}(j \omega)=\frac{R_{0}(\rho+1)^{2}}{2 \underline{Z}+R_{0}(2 \rho-T+2)}, \\
\underline{S}_{21}(j \omega)=\frac{2 T \underline{Z}+R_{0}(\rho+1)^{2}}{2 \underline{Z}+R_{0}(2 \rho-T+2)},
\end{gathered}
$$

where

$$
\underline{Z}=R+j L \omega
$$

Therefore, the transmission phase $\varphi(\omega)=\angle \underline{S}_{12}(j \omega)$ is expressed as: 


$$
\begin{aligned}
& \varphi(\omega)=\arctan \left[\frac{2 T \cdot L \omega}{2 T \cdot R+R_{0}(\rho+1)^{2}}\right], \\
& -\arctan \left[\frac{2 L \omega}{2 R+R_{0}(2 \rho-T+2)}\right]
\end{aligned}
$$

We find that at very low frequencies $(\omega \approx 0)$, these Sparameters become:

$$
\begin{gathered}
S_{11}(0)=S_{22}(0)=\frac{R_{0}(T-1)+\rho\left(2 R+\rho \cdot R_{0}\right)}{2 R+R_{0}(2 \rho-T+2)}, \\
S_{12}(0)=\frac{R_{0}(\rho+1)^{2}}{2 R+R_{0}(2 \rho-T+2)}, \\
S_{21}(0)=\frac{2 T \cdot R+R_{0}(\rho+1)^{2}}{2 R+R_{0}(2 \rho-T+2)} .
\end{gathered}
$$

According to relation (13), the NGD cell requires the following stability condition:

$$
\left|S_{11}(0)\right|<1 \quad \Leftrightarrow \quad R>R_{\min }=R_{0} \frac{\rho^{2}-2 \rho+2 T-3}{2(1-\rho)} .
$$

Moreover, it can be demonstrated from equation (10) that at very low frequencies $(\omega \approx 0)$, the group delay of the circuit which is analytically defined as:

$$
\tau(\omega)=-\frac{\partial \angle \underline{S}_{21}(j \omega)}{\partial \omega},
$$

is expressed as:

$$
\tau(0)=\frac{2 L \cdot R_{0}(\rho-T+1)^{2}}{\left[2 R+R_{0}(2 \rho-T+2)\right]\left[R_{0}(\rho+1)^{2}+2 T \cdot R\right]} .
$$

It means that to generate NGD with the circuit introduced in Figure 1 at very low frequencies $(\tau(0)<0)$, the following condition must be satisfied:

$$
2 R+R_{0}(2 \rho-T+2)<0 \Leftrightarrow R<(T / 2-\rho-1) R_{0} .
$$

\subsubsection{Synthesis relations}

At the beginning of the synthesis process, the used amplifier is supposed characterized by its S-parameter elements $T$ and $\rho$. Thus, from the desired values of gain $\left|S_{21}(0)\right|$ and NGD $\tau(0)$, the synthesis relations enabling to determine the NGD circuit parameters $R$ and $L$ are extracted by inverting relations (15) and (18) respectively. From where are established the following formulations [16]:

$$
\begin{aligned}
& R=R_{0} \frac{S_{21}(0)(T-2 \rho-2)+(\rho+1)^{2}}{2\left[S_{21}(0)-T\right]}, \\
& L=\tau(0) \frac{\left\{\begin{array}{l}
{\left[2 R+R_{0}(2 \rho-T+2)\right]} \\
{\left[R_{0}(\rho+1)^{2}+2 T \cdot R\right]}
\end{array}\right\}}{2 R_{0}(\rho-T+1)^{2}} .
\end{aligned}
$$

This expression is dedicated to the synthesis with $\tau(0)$ assigned as a real negative. Under the following condition:

$$
T>\rho+2,
$$

the result of expression (20) is physically meaningful only under the following condition:

$$
S_{21}(0)>S_{21 \min }(0)=\frac{(\rho+1)^{2}}{\rho-T+2} .
$$

\subsubsection{Fundamental characteristics of the baseband NGD cell understudy}

One of the main properties which characterizes the NGD cell understudy is based on the relation between its transmission parameter $S_{21}(0)$ and its reflection parameters $S_{11}(0)=S_{22}(0)$. In fact, giving the transmission parameter $T$ and the return loss $\rho$ of the amplifier A, one demonstrates that the return losses $S_{11}(0)=S_{22}(0)$ are mathematically linked to the insertion gain $S_{21}(0)$ by the relation:

$$
S_{11}(0)=S_{22}(0)=\frac{(\rho+1)\left[\rho+1-S_{21}(0)\right]+T}{\rho-T+1} .
$$

To achieve the basic stability condition:

$$
\left|S_{11}\right|=\left|S_{22}\right|<1
$$

once again, the transmission parameter $S_{21}(0)$ must verify inequality (23). Furthermore, under condition (19), the group delay $\tau(\omega)$ of the NGD cell can be negative from $\omega \approx 0$ to the cut-off frequency $\omega_{c}$. Via the derivation of the transmission phase with respect to $\omega$, it is established that this cut-off frequency is expressed as:

$$
\omega_{c}=\frac{\sqrt{\left[(\rho+1)^{2} R_{0}+2 T \cdot R\right]}}{2 L \sqrt{T}} .
$$

In order to verify the relevance of the above theoretic concept, in the next section, we will examine the Sparameters and the group delay of a proof of concept device designed with the NGD topology proposed. 


\subsection{Numerical demonstration of the negative time effects}

The simulation results presented in this section were generated from the well-known the commercial software Advanced Design system (ADS) from Agilent ${ }^{\mathrm{TM}}$.

\subsubsection{Design of the prototype of base band NGD device tested}

As illustrated in Figure 2, a circuit composed of two-stage NGD cells was designed and simulated. After the application of the previous synthesis relations accompanied with a slight optimization of the RF-components, the choice of the relevant nominal values of passive elements were carried out.

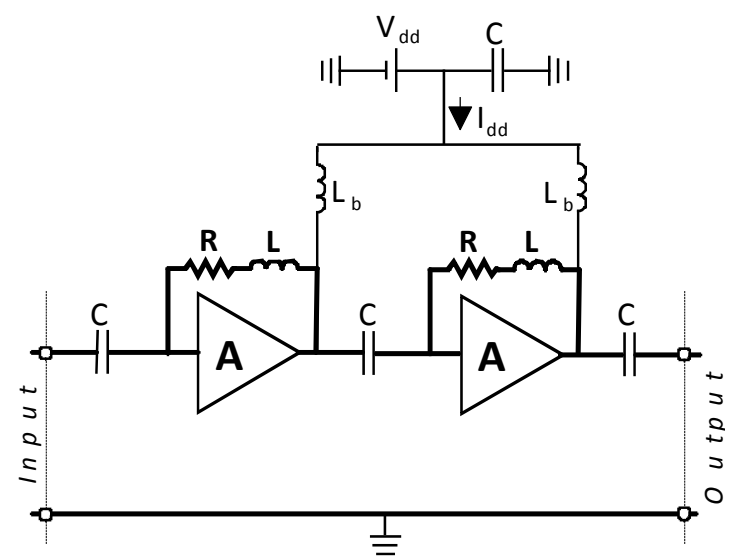

Figure 2: Prototype of two-stage NGD circuit for testing the time advance.

Then, comparative simulations were performed between the circuit comprised of ideal RF-part and the complete circuit shown in Figure 2. This latter was simulated with the Sparameter realistic models of passive components $\mathrm{R}, \mathrm{L}$ and $\mathrm{C}$ available in ADS library. Table 1 indicates the references of the components employed. The employed amplifiers are MMIC LNAs HMC 395 provided by HITTITE ${ }^{\mathrm{TM}}$. In this study, the amplifiers were biased with the DC voltage $V_{d d}=$ $5 \mathrm{~V}$ and $I_{d d}=20 \mathrm{~mA}$

The considered MMIC LNAs were replaced by the measured S-parameters including the bias networks provided by the manufacturer.

Table 1: References of the passive components constituting the circuit shown in Figure 2.

\begin{tabular}{cccc}
\cline { 2 - 4 } & $\begin{array}{c}\text { Nominal } \\
\text { value }\end{array}$ & Reference & Manufacturer \\
\hline Resistance & $R=27 \Omega$ & $\begin{array}{c}\text { AVX CR } \\
10-270 \mathrm{~J}\end{array}$ & AVX \\
\hline \multirow{2}{*}{ Inductance } & $L=100 \mathrm{nH}$ & $\begin{array}{c}\text { LQG15HSR } \\
10 \mathrm{~J} 02\end{array}$ & MURATA \\
\cline { 2 - 4 } & $L_{b}=100 \mu \mathrm{H}$ & $\begin{array}{c}\text { AIC1210- } \\
101 \mathrm{~J}\end{array}$ & COILCRAFT \\
\hline Capacitor & $C=1 n F$ & - & MURATA \\
\hline
\end{tabular}

\subsubsection{Frequency domain investigations}

One points out that the simulation results presented in this paragraph were realized by considering first the ideal RF circuit and then, the realistic circuit shown in Figure 2 by considering the touchstone or $\mathrm{S}_{2} \mathrm{P}$ (or two-ports $\mathrm{S}$ parameter) models of the passive components provided by the manufacturer. According to the frequency bands of the components utilized, S-parameter simulations were performed. As displayed in Figure 3, a good agreement between the frequency simulations with the ideal and realistic circuits is found in the NGD frequency band.

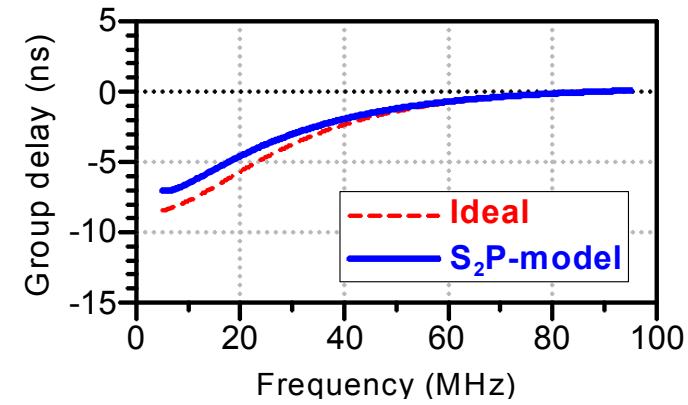

Figure 3: Comparison of the group delay frequency responses from ideal RF circuit and realistic circuit with S2P models including the bias networks.

As forecasted in theory, group delay presenting negative values were realized up to about $90 \mathrm{MHz}$. This NGD value goes down to about $-7 \mathrm{~ns}$ at very low frequencies $(\omega \approx 0)$. As shown in Figure 4, the circuit simulated guarantees an average gain more than $0 \mathrm{~dB}$ from very low frequencies up to hundreds $\mathrm{MHz}$.

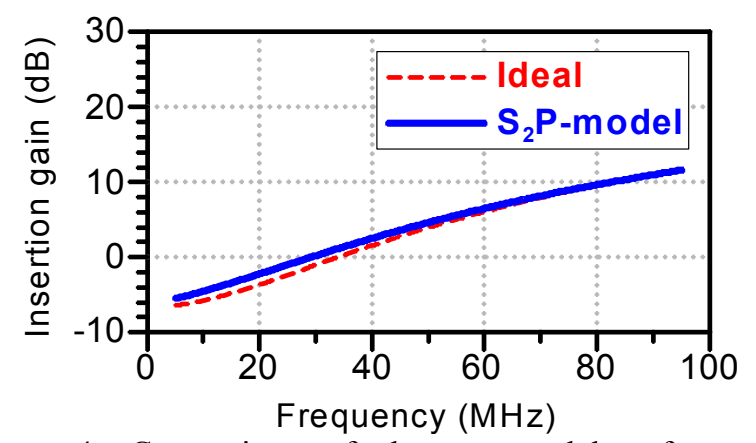

Figure 4: Comparison of the group delay frequency responses from the ideal RF circuit and realistic circuit with S2P models including the bias networks.

\subsubsection{Stability analysis}

The access parameters $S_{11}$ and $S_{22}$ of the circuit tested are plotted in Figure 5. We remark that these parameters confirm the stability of the circuit from DC to $5 \mathrm{GHz}$. Moreover, in this ultra wide frequency band, the isolation parameter $S_{12}$ is better than $-20 \mathrm{~dB}$. To strengthen the stability of the tested device, the stability factor was also examined. One recalls that this factor is defined by: 


$$
\mu(\omega)=\frac{1-\left|S_{11}(j \omega)\right|^{2}}{\left|S_{22}(j \omega)-S_{11}^{*}(j \omega) \Delta(j \omega)\right|+\left|S_{12}(j \omega) S_{21}(j \omega)\right|},
$$

with

$$
\Delta(j \omega)=S_{11}(j \omega) S_{22}(j \omega)-S_{12}(j \omega) S_{21}(j \omega),
$$

and $S_{11}^{*}(j \omega)$ is the conjugate of $S_{11}(j \omega)$.

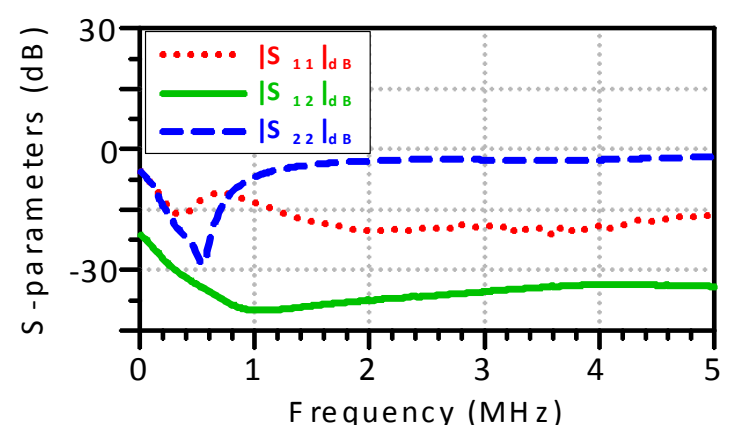

Figure 5: Isolation and return losses of the NGD device shown in Figure 2.

As reported in [17], this behaviour of the transmission parameter magnitude can be exploited to generate the pulse compression effect. The Rollet factor of the circuit is also plotted in Figure 6.

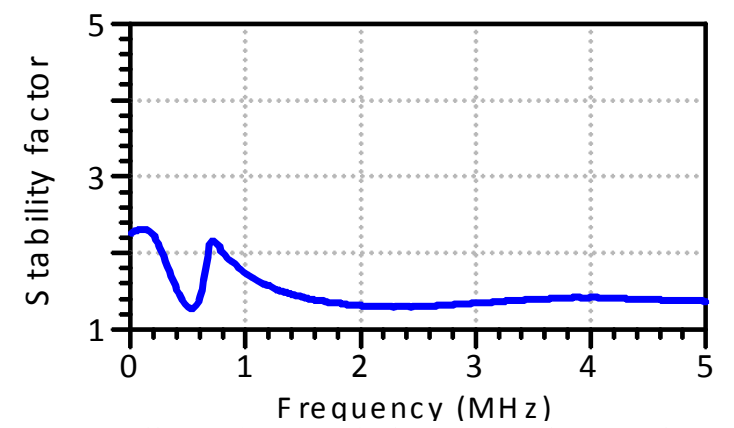

Figure 6: Rollet's factor of the NGD device shown in Figure 2 .

As conclusion, the NGD topology proposed in this section respects all the constraints of classical RF active devices as the stability condition in UWB frequency band.

\subsubsection{Time-domain investigations}

For the better understanding about the meaning of the NGD effect generated by the circuit understudy, time-domain simulations were also performed in the transient option of SPICE-ADS environment. Figure 7 displays the configuration of the simulated circuit which is loaded by $R_{0}$ $=50 \Omega$. After ADS time-domain simulations, the results plotted in Figure 8 and Figure 9 were obtained.

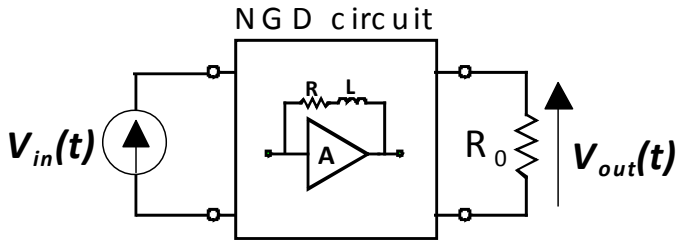

Figure 7: NGD circuit shown in Figure 2 loaded by $R_{0}=50$ $\Omega$.

We can see that as explained by Figure 8 , the output $V_{\text {out }}$ of the NGD circuit is practically conform to the Gaussian input $V_{\text {in }}$ which presents here a half height half width of about 18 ns. Moreover, contrarily to the ordinary circuit transient response, thanks to the NGD phenomenon, the wave fronts of the output $V_{\text {out }}$ is slightly in advance of about $7 \mathrm{~ns}$ compared to the input $V_{i n}$.

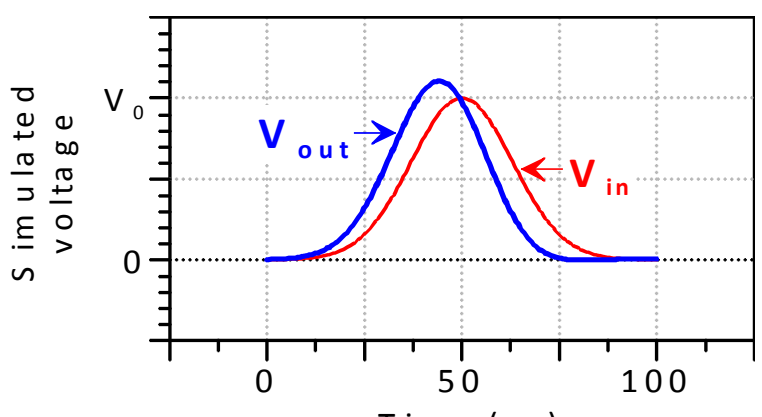

Time (ns)

Figure 8: NGD circuit transient analysis results.

In other hand, by injecting an arbitrary waveform input signal presented by $V_{\text {in }}$ of Figure 9, once again, an effect of the negative time-delay with time-shift of about $7 \mathrm{~ns}$ is found.

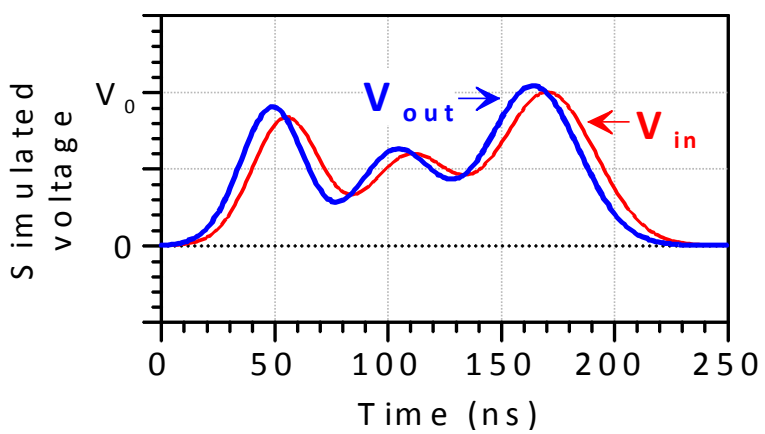

Figure 9: NGD circuit transient analysis results.

Despite this outstanding effect, it is interesting to note that the output signal appears after the input at the starting point $t=0 \mathrm{~s}$. So, the causality principle is still respected. As reported in [18], this advance phenomenon occurs only when the input spectrum belongs in the NGD frequency band.

\subsection{Comments and discussions}

An innovative NGD active circuit topology susceptible to operate at baseband frequencies is developed by using a microwave amplifier. Compared to the NGD topologies 
introduced in [14], the proposed one presents a facility to achieve a good access matching and enables to avoid the opposite sign between the output and input voltages for the single or odd number of stage circuit. Theoretical approaches based on the S-parameter analysis were proposed. Then, the synthesis relations enabling to design the electronic device with the NGD topology understudy were analytically demonstrated according to the desired values of the gain and NGD. Then, the theoretic characteristic specific to the circuit analyzed vis-à-vis the NGD effects were also established. To illustrate the relevance of the theoretic principle proposed, design and simulations of two-stage circuit based on the use MMIC LNA device were carried out. So, it demonstrates the possibility to generate NGD simultaneously with gain in baseband up to microwave frequencies. Moreover, it was stated with SPICE simulation that the presented NGD topology respects all the criteria of classical active $\mathrm{RF}$ circuits.

Furthermore, it was shown also that the NGD circuit investigated can be employed to reduce the pure delay induced by telecommunication devices as the microwave TLs. As application, this NGD circuit is potentially useful for the delay reduction as proposed in [37-40] notably for the correction of interconnections degradation on the PCB and for the high-speed microelectronic systems as illustrated in $[41-43]$.

\section{Examination of the NGD circuit formed by an LNA for RF/microwave applications}

Figure 10 presents the microwave NGD circuit using LNA understudy in the present section. This unit cell of the understudy NGD circuit topology is composed of an amplifier in cascade with an RLC-series resonant network.

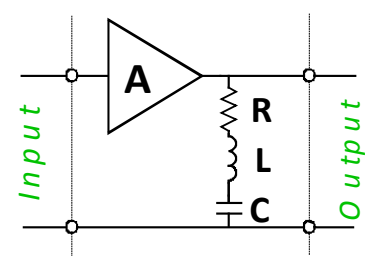

Figure 10: Microwave amplifier cascaded with a shunt series RLC resonant network.

\subsection{Theory on the NGD circuit operating in $\mathrm{RF} / \mathrm{microwave}$ frequencies}

Similar to the previous section, to examine the functioning of the circuit described in Figure 10, we analyse its Sparameters and establish the synthesis relations enabling to determine the elements of the NGD topology in function of the desired NGD and gain values. Then, properties of the topology will be established.

\subsubsection{S-parameters analysis}

Through the S-parameter theory, by considering the reference impedance $R_{0}$, one demonstrates that the $\mathrm{S}$ parameters of the NGD topology presented in Figure 10 are expressed as:

$$
\begin{gathered}
S_{11}=\rho, \\
S_{12}=0, \\
\underline{S}_{21}=\frac{2 T \cdot \underline{Z}}{R_{0}(\rho+1)+2 \underline{Z}}, \\
\underline{S}_{22}=\frac{2 \rho \cdot \underline{Z}-R_{0}(\rho+1)}{R_{0}(\rho+1)+2 \underline{Z}} .
\end{gathered}
$$

where

$$
\underline{Z}=R+j L \omega+\frac{1}{j C \omega}
$$

is the impedance of the series RLC resonant network. The transmission phase of this microwave NGD topology is expressed as:

$$
\varphi(\omega)=\arctan \left(L \omega-\frac{1}{C \omega}\right)-\arctan \left[\frac{2\left(L \omega-\frac{1}{C \omega}\right)}{2 R+R_{0}(\rho+1)}\right]
$$

One demonstrates that at the resonance frequency:

$$
\omega_{0}=\frac{1}{\sqrt{L \cdot C}}
$$

the transmission parameter $S_{21}$ and the output return loss $S_{22}$ become respectively:

$$
\begin{aligned}
& S_{21}\left(\omega_{0}\right)=\frac{2 T \cdot R}{R_{0}(\rho+1)+2 R}, \\
& S_{22}\left(\omega_{0}\right)=\frac{2 \rho \cdot R-R_{0}(\rho+1)}{R_{0}(\rho+1)+2 R} .
\end{aligned}
$$

Similarly to the NGD cell introduced in [14-16], the group delay will be also always negative at this resonance frequency:

$$
\tau\left(\omega_{0}\right)=\frac{-2 L \cdot R_{0}(\rho+1)}{R\left[R_{0}(\rho+1)+2 R\right]}
$$

\subsubsection{Properties of the NGD circuit understudy}

One can establish also that this microwave NGD value belongs in the frequency band corresponding to 
$\left[\omega_{1}, \omega_{2}\right]$ which is delimited by the following cut off frequencies:

$$
\begin{aligned}
& \omega_{1}=\sqrt{\frac{4 L+L_{0}-\sqrt{L_{0}\left(8 L+L_{0}\right)}}{4 L^{2} C}}, \\
& \omega_{2}=\sqrt{\frac{4 L+L_{0}+\sqrt{L_{0}\left(8 L+L_{0}\right)}}{4 L^{2} C}} .
\end{aligned}
$$

where

$$
L_{0}=R \cdot C\left[R_{0}(\rho+1)+2 R\right]
$$

It is interesting to note that the product of these NGD cut-off frequencies verify the following relation:

$$
\omega_{1} \cdot \omega_{2}=\omega_{0}^{2}
$$

But one points out that at very low frequencies $\omega \approx 0$, the group delay is always positive.

$$
\tau(0)=\frac{C \cdot R_{0}(\rho+1)}{2}
$$

This last expression leads us to the synthesis method of the NGD passive elements in the next paragraph.

\subsubsection{Synthesis method}

By inverting formulae (34) and (36) and the resonance frequency expression $\omega_{0}$, it yields the following synthesis relations:

$$
\begin{gathered}
R=\frac{S_{21} R_{0}(\rho+1)}{2\left(T-S_{21}\right)}, \\
L=-\frac{\tau\left|S_{21}\right| R_{0}(\rho+1-T)^{2}}{2\left(T+\left|S_{21}\right|\right)^{2}}, \\
C=\frac{1}{L \cdot \omega_{0}^{2}} .
\end{gathered}
$$

In this case, the output return loss $S_{22}$ will be systematically fixed to:

$$
S_{22}\left(\omega_{0}\right)=\frac{\rho+1}{T}\left|S_{21}\left(\omega_{0}\right)\right|-1 .
$$

Of course, for one cell, the gain $S_{21}$ can not be greater than $T$. Moreover, to provide a significant NGD with amplification in the wide bandwidth, the investigation of the cascaded structure is provided in the next subsection.
In order to verify the efficiency of the proposed theory, analysis of validation results will be proposed in the next section.

\subsection{Numerical validation of the proposed NGD circuit for the modulated signals}

The present design and simulation results were performed by using the microwave and electronic circuit simulator Advanced Design System (ADS) from Agilent ${ }^{\mathrm{TM}}$.

\subsubsection{Design of the microwave NGD device tested}

The poof of concept to validate the former theoretic approach is comprised of the one-stage NGD active circuit shown in Figure 11. This later is designed with the LNA implemented in non-linear model using the package transistor AT-41411 provided by Agilent ${ }^{\mathrm{TM}}$. Moreover, the considered passive components are from passive SMT models available in ADS library.

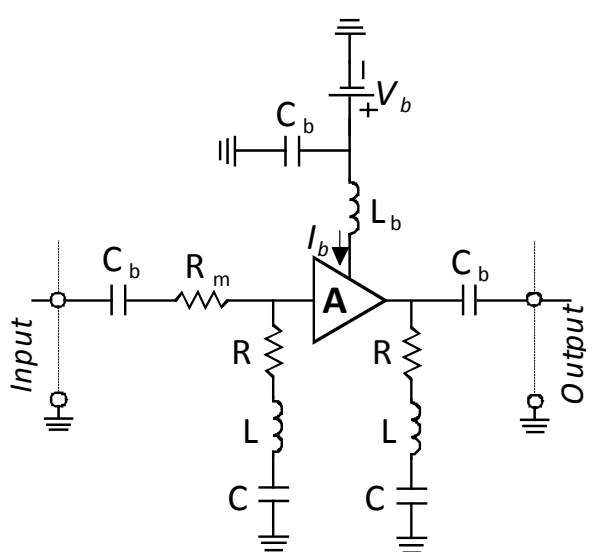

Figure 11: Simulated one stage NGD circuit. $\left(R=36 \Omega, R_{m}\right.$ $\left.=22 \Omega, L=33 \mathrm{nH}, C=0.5 \mathrm{pF}, L_{b}=220 \mathrm{nH}, C_{b}=10 \mathrm{nF}\right)$.

The tested NGD device is biased at the DC voltage $V_{b}=9 \mathrm{~V}$ and $I_{b}=3.9 \mathrm{~mA}$.

\subsubsection{Frequency-domain analysis}

Through the electromagnetic (EM) and circuit cosimulations in SPICE-Momentum ADS environment, the results obtained are sketched in Figure 12 and Figure 13. As illustrated in Figure 12, the tested circuit introduced in Figure 11 enables to generate a narrow-band NGD from $1.09 \mathrm{GHz}$ to $1.26 \mathrm{GHz}$ which can go down below $-1.5 \mathrm{~ns}$. 


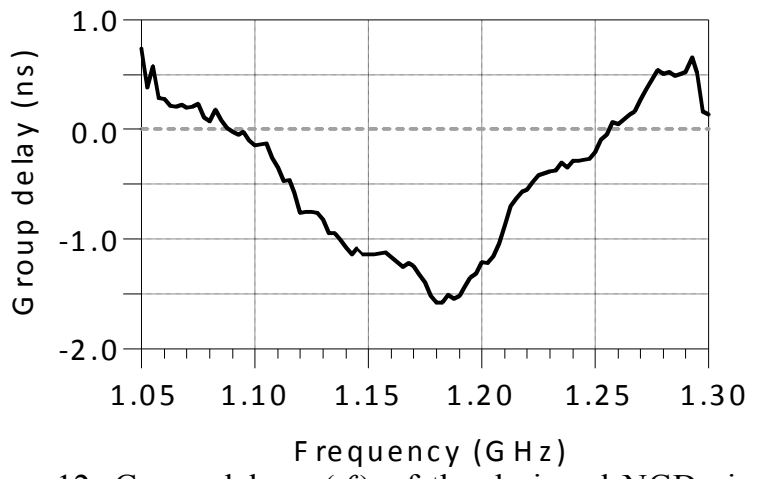

Figure 12: Group delay $\tau(f)$ of the designed NGD circuit tested.

Thus, one evaluates over the bandwidth of about $170 \mathrm{MHz}$. As displayed in Figure 13, in the considered frequency band, the insertion gain is above $0.5 \mathrm{~dB}$ and a good matching at the two accesses is achieved.

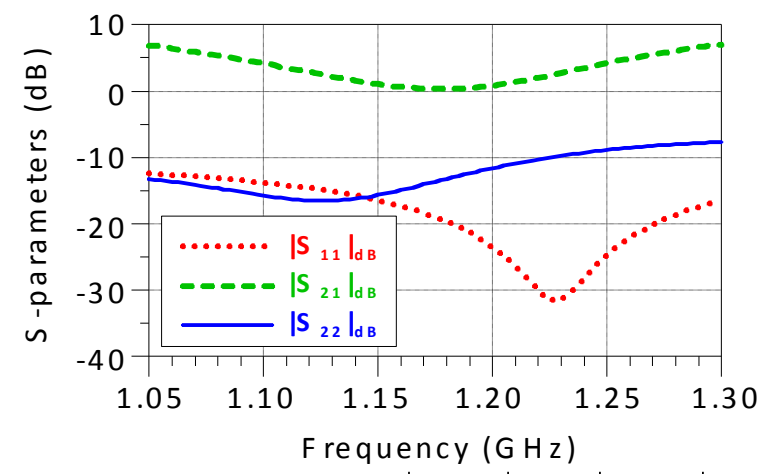

Figure 13: S-parameters $\left(\left|S_{11}(f)\right|_{d B},\left|S_{21}(f)\right|_{d B}\right.$ and $\left|S_{22}(f)\right|_{d B}$ ) of the designed NGD circuit shown in Figure 11.

\subsubsection{Analysis of the proposed NGD device non-linearity}

To carry out a non-linear analysis of the circuit shown in Figure 11, harmonic balance simulations with the considerations of harmonics up to $11^{\text {th }}$ order were realized in the SPICE schematic environment of ADS. For that, a single tone harmonic signal with power $P_{i n_{-} d B m}$ at the single frequency $f_{0}=1.18 \mathrm{GHz}$ and varied from $-15 \mathrm{dBm}$ to 10 $\mathrm{dBm}$ was injected in the circuit tested. As consequence, the plot of the output power $P_{\text {out } d B m}$ are obtained in function of the input level $P_{i \_n d B m}$ depicted in Figure 14.

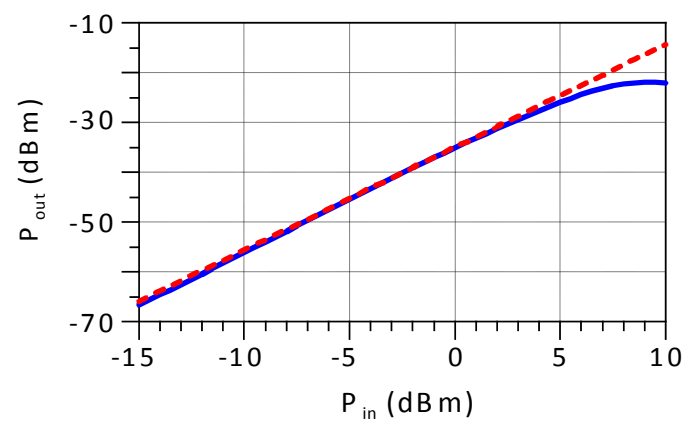

Figure 14: Harmonic balance simulation results: $P_{\text {out } d B m}$ vs $P_{\text {indBm. }}$.

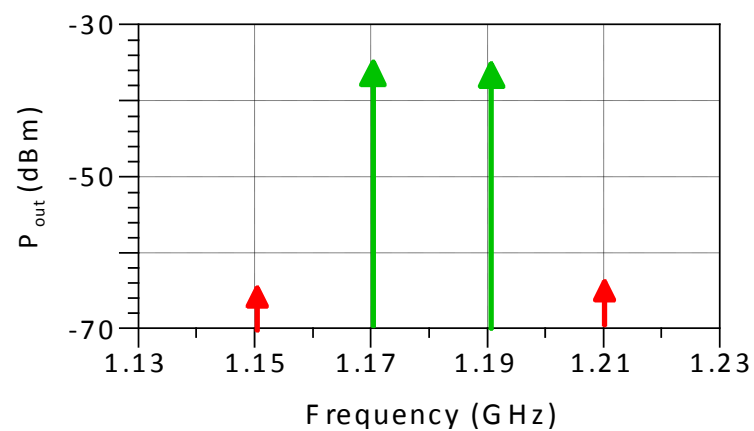

Figure 15: Third order intermodulation products $\mathrm{IMD}_{3}$ of the device shown in Figure 11.

So, one can see that the device understudy presents $P_{\text {inldBC }}$ of about $5 \mathrm{dBm}$. Furthermore, by injecting two tones signal centred at the NGD centre frequency $f_{0}=1.19 \mathrm{GHz}$ with frequency shift of about $20 \mathrm{MHz}$. The input powers $P_{\text {in }} d B m$ of these signals are set at around $5 \mathrm{dBm}$. After simulations, the result obtained is displayed in Figure 15.

Due to the non-linearity of the considered NGD circuit, for the first order harmonic with frequencies $f_{1}=1.17 \mathrm{GHz}$ and $f_{2}=1.19 \mathrm{GHz}$, it appears $P_{\text {out }}\left(1^{\text {st }}\right.$ order $) \approx-31 \mathrm{dBm}$ and $P_{\text {out }}\left(3^{\text {rd }}\right.$ order $) \approx-65 \mathrm{dBm}$ in the operating frequency band.

\subsection{Discussions}

A microwave topology of active circuit susceptible to exhibit the NGD function with modulated signals is proposed in this section. The NGD topology investigated is comprised of an LNA in cascade with a shunt RLC-series network. The analysis of the proposed NGD topology Sparameters was conducted. The theoretic concept is then, completed by the establishment of synthesis methods of the NGD circuit parameters. This synthesis must be realized knowing the characteristics of the used amplifier and also the desired values of gain and NGD at the given specific frequency. The relevance of the theory was confirmed by the simulation results of one-stage NGD device designed with a hybrid LNA based on the package transistor AT41411 from Agilent ${ }^{\mathrm{TM}}$. Against the NGD active topology proposed in [15-18], through the present study, it is proved that a better access matching easily is achieved with a simpler biasing network.

\section{General conclusions}

A theory on the active topologies of electronic circuit susceptible to generate the NGD function is successfully investigated in this paper. Two different types of active topology were analyzed and synthesized.

The first topology, mainly dedicated to the base band frequencies, is comprised of an RL-passive network in 
feedback with an RF/microwave amplifier. The other one, mainly operating with the modulated $\mathrm{RF} /$ microwave signals, is formed by an RLC-passive network associated in cascade with the amplifier. Theoretical analyses based on the S-parameters are presented by considering the gain and the matching access of the amplified used. Then, synthesis formulae for determining the elements $\mathrm{R}, \mathrm{L}$ and $\mathrm{C}$ of the NGD circuits in function of the desired values of the gain and NGD are established. In addition, the basic properties of the NGD topologies investigated were also proposed. For checking the validity of the theoretical approaches, simulations both in frequency- and time-domains were performed.

With the base band NGD topology, NGD from DC up to tens $\mathrm{MHz}$ were realized accompanied with loss compensation. It was shown that the matching access levels of the proof of concept were widely lower than $0 \mathrm{~dB}$ up to 5 $\mathrm{GHz}$. It was stated that the tested NFD circuit is unconditionally stable. Then, time-domain simulations confirm the functioning of the NGD circuit. It was observed that by injecting arbitrary wave form signals to the NGD circuit, it was demonstrated that the output signal wave fronts exit the circuit before those of the input penetrating in the circuit. As argued in [10-14, 19-20], this effect is not in contradiction with the causality because the input bandwidth is sufficiently limited. Whereas with the second NGD topology investigated, NGD around $1.19 \mathrm{GHz}$ with minimal value of about $-1.5 \mathrm{~ns}$ was generated. It is shown that the proof of concept circuit presents good level of matching and excellent isolation loss. Finally, non-linear analyses were proposed to show the reaction of the NGD circuit with the power of the operating signals.

In the continuation of this work, the NGD topologies investigated will be integrated in the telecommunication systems for the enhancement of architectures notably for the delay cancellation.

As ongoing research, design of circuit using MMIC LNA operating with base band signals up to $\mathrm{RF} /$ microwave frequencies is planned. The design of the NGD device capable to work up to several tens $\mathrm{GHz}$ based on distributed elements is also in progress. Lastly, the NGD concept is envisaged to be used for reducing the delays and also for enhancing the radio communication systems as reported in [45-48].

\section{Acknowledgements}

Acknowledgement is made to European Union (EU) and Upper Normandy region (FRANCE) for the support of this research through the European Interreg IVA project No 4081 entitled "Time-Domain Electromagnetic Characterization and Simulation (TECS)".

\section{References}

[1] S. Lucyszyn, I. D. Robertson and A. H. Aghvami, "Negative group delay synthesiser", Electronic Letters, Vol. 29 , pp. 798-800, 1993
[2] S. Lucyszyn, "Lies, damn lies and measurements," in Proc. of 16th APMC E4, New Delhi, India, Dec. 2004.

[3] C. D. Broomfield and J. K. A. Everard, "Broadband negative group delay networks for compensation of oscillators using feedforward amplifiers", Electronics Letters, Vol. 20, 1710-1711, Sep. 2000.

[4] G. V. Eleftheriades, O. Siddiqui and A. K. Iyer, "Transmission line for negative index media and associated implementations without excess resonators," IEEE MWC Lett., Vol. 13, No. 2, pp. 51-53, Feb. 2003.

[5] O. F. Siddiqui, M. Mojahedi, and G. V. Eleftheriades, "Periodically loaded transmission line with effective negative refractive index and negative group velocity," IEEE Trans. Ant. Prop., Vol. 51, No. 10, pp. 2619-2625, Oct. 2003.

[6] N. S. Bukhman and S. V. Bukhman, "On the negative delay time of a narrow-band signal as it passes through the resonant filter of absorption," Radiophysics and Quantum Electronics, Vol. 47, No. 1, pp. 66-76, 2004.

[7] O. F. Siddiqui, M. Mojahedi and G. V. Eleftheriades, "Periodically Loaded Transmission Line With Effective Negative Refractive Index and Negative Group Velocity", IEEE Transactions on Antennas and Propagation, Vol. 51, No. 10, pp. 2619-2625, Oct. 2003.

[8] J. F. Woodley and M. Mojahedi, "Negative Group Velocity and Group Delay in Left-Handed Media", Phys. Rev. E, Vol. 70, pp. 046603.1-046603.6, 2004.

[9] R. Y. Chiao, E. L. Bolda, J. Bowie, J. Boyce and M. W. Mitchell, "Superluminality and Amplifiers," Prog. Crystal Growth Charact. Mat. 33, pp. 319-325, 1996.

[10] D. Solli, and R. Y. Chiao, "Superluminal effects and negative delays in electronics, and their applications," Phys. Rev. E, No. 5, Nov. 2002.

[11] M. Kitano, T. Nakanishi and K. Sugiyama, "Negative group delay and superluminal propagation: An electronic circuit approach," IEEE J. Selected Topics in Quantum Electronics, Vol. 9, No. 1, pp. 43-51, 2003.

[12] J. N. Munday and R. H. Henderson, "Superluminal time advance of a complex audio signal", Appl. Phys. Lett., Vol. 85, pp. 503-504, Jul. 2004.

[13] S. J. Erickson, M. Khaja and M. Mojahedi, "Timeand frequency-domain measurements for an active negative group delay circuit", in Proc of IEEE Ant. Prop. Soc. Int. Symp., Vol. 3A, pp. 790-793, 2005.

[14] B. Ravelo, "Demonstration of negative signal delay with short-duration transient pulse", Eur. Phys. J. Appl. Phys. (EPJAP), Vol. 55, 10103-pp. 1-8, 2011.

[15] B. Ravelo and S. De Blasi, "An FET-based microwave active circuit with dual-band negative group delay", Journal of Microwaves, Optoelectronics and Electromagnetic Applications (JMOe), Vol. 10, No. 2, pp. 355-366, Dec.2011.

[16] B. Ravelo, "Investigation on microwave negative group delay circuit", Electromagnetics, Vol. 31, No. 8, pp. 537-549, Nov. 2011. 
[17] B. Ravelo, A. Perennec, M. Le Roy and Y. Boucher, "Active microwave circuit with negative group delay," IEEE MWC Lett., Vol. 17, No. 12, pp. 861-863, Dec. 2007.

[18] B. Ravelo, A. Perennec and M. Le Roy, "Synthesis of broadband negative group delay active circuits," IEEE MTT-Symposium IMS 2007, Honolulu, Hawaii, pp. $2177-$ 2180, 3-8 June 2007.

[19] M.W. Mitchell and R.Y. Chiao, "Causality and negative group delays in a simple bandpass amplifier", $\mathrm{Am}$. J. Phys. 66, 14-19, 1998.

[20] M.W. Mitchell and R.Y. Chiao, "Negative group delay and 'Fronts' in a causal systems: An experiment with very low frequency bandpass amplifiers", Phys. Lett., Vol. A230, 133-138, June 1997.

[21] M. Kandic and G. E. Bridges, "Asymptotic Limits of Negative Group Delay in Active Resonator-Based Distributed Circuits", IEEE Transactions on Circuits and Systems I: Regular Papers, Vol. 58, No. 8, pp. 1727 - 1735, Aug. 2011.

[22] M. Kandic and G. E. Bridges, "Transient-Imposed Limitations of Negative Group Delay Circuits", in Proc. of 14th International Symposium on Antenna Technology and Applied Electromagnetics \& the American Electromagnetics Conference (ANTEM-AMEREM), 2010, Ottawa, ON, Canada, pp. 1-4, 5-8 July 2010.

[23] A. Dogariu, A. Kuzmich and L. J. Wang, "Transparent anomalous dispersion and superluminal lightpulse propagation at a negative group velocity", Phys. Rev. $A$, Vol. 63, pp. 053806.1-053806.12, 2001.

[24] L. Brillouin and A. Sommerfeld, Wave propagation and group velocity, Academic Press, New York, 113-137, 1960.

[25] G. B. Garrett and D. E. McGumber, "Propagation of a Gaussian light pulse through an anomalous dispersion medium", Phys. Rev. A, Vol. 1, pp. 305-313, 1970.

[26] S. Chu. and S. Wong, "Linear pulse propagation in an absorbing medium", Phys. Rev. Lett., Vol. 48, pp. 738-741, 1982.

[27] Ségard and B. Macke, "Observation of negative velocity pulse propagation”, Phys. Lett. 109, pp. 213-216, 1985.

[28] B. Ravelo, M. Le Roy and A. Perennec, "Application of negative group delay active circuits to the design of broadband and constant phase shifters", Microwave and Optical Technology Letters, Vol. 50, No. 12, pp. 3077-3080, Dec. 2008.

[29] S. Keser and M. Mojahedi, "Broadband negative group delay microstrip phase shifter design", in Proc. of IEEE Ant. Prop. Soc. Int. Symp. 2009 (APSURSI'09), Charleston, SC, pp. 1-4, 1-5 June 2009.

[30] B. Ravelo, A. Perennec and M. Le Roy, "Synthesis of frequency independent phase shifter using negative group delay active circuit," Int. J. RF and Microwave ComputerAided Engineering (RFMiCAE), Vol. 21, No. 1, pp. 17-24, Jan. 2011.
[31] B. Ravelo, M. Le Roy and A. Pérennec, "Frequency-independent active phase shifters for UWB applications", Proc. of the 40th European Microwave Conference, Paris, France, pp. 1774-1777, 28-30 Sep. 2010.

[32] B. Ravelo, A. Perennec and M. Le Roy, "Broadband balun using active negative group delay circuit", in Proc. of 37th European Microwave Conference, Munich, Germany, pp. 466-469, Oct. 2007.

[33] H. Noto, K. Yamauchi, M. Nakayama, and Y. Isota, "Negative group delay circuit for feed-forward amplifier", IEEE Int. Microw. Symp. Dig., Honolulu, Hawaii, pp. 1103-1106, Jun. 2007.

[34] H. Choi, Y. Jeong, C. D. Kim, and J. S. Kenney, "Bandwidth enhancement of an analog feedback amplifier by employing a negative group delay circuit", Progress In Electromagnetics Research, Vol. 105, 253-272, 2010.

[35] B. Ravelo, "Investigation on the microwave pulse signal compression with NGD active circuit", PIER $C$ Journal, Vol. 20, pp. 155-171, 2011.

[36] B. Ravelo, Étude des circuits analogiquesnumériques NGD et leurs applications: Théorie fondamentale et expérimentations des circuits analogiques et numériques à temps négatif et leurs applications, (in French) Chapters 8, Éditions Universitaires Européennes (EUE), Ed. by M. Fabre, 432 Pages, 13 Jan. 2012.

[37] B. Ravelo and J. Ben Hadj Slama, "Equalization of digital/mixed-signal disturbances with an negative group delay circuit", (Accepted for communication) Proceedings of the 16th IEEE Mediterranean Electrotechnical Conference (MELECON 2012), 25-28 March 2012, Medine Yasmine Hammamet, Tunisia.

[38] T. Eudes and B. Ravelo, "Cancellation of delays in the high-rate interconnects with UWB NGD active cells", Applied Physics Research, Vol. 3, No. 2, Nov. 2011, pp. 8188.

[39] B. Ravelo, A. Perennec and M. Le Roy, "Experimental validation of the RC-interconnect effect equalization with negative group delay active circuit in planar hybrid technology", in Proc. of 13th IEEE Workshop on Signal Propagation Interconnects (SPI), Strasbourg, France, May 2009.

[40] B. Ravelo, A. Perennec and M. Le Roy, "New technique of inter-chip interconnect effects equalization with negative group delay active circuits", VLSI, Chap. 20, Ed. by Z. Wang, INTECH book, 409-434, Feb. 2010.

[41] B. Ravelo, "Baseband NGD circuit with RF amplifier”, Electronic Letters, Vol. 47, No. 13, June 2011.

[42] B. Ravelo, "Delay modelling of high-speed distributed interconnect for the signal integrity prediction", To be published in Eur. Phys. J. Appl. Phys. (EPJAP), 2012, DOI: 10.1051/epjap/20121103742012.

[43] B. Ravelo, "Lumped RC-tree network propagationdelay modeling", To be published in Int. J. Communication Engineering Applications (IJCEA), 2012. 
[44] R. Mumford, "European carriers face capacity crunch challenges", Int. Report, Microwave J., 169-170, Apr. 2010.

[45] A. Lazaro, D. Girbau and R. Villarino, "Effects of interferences in UHF RFID systems", Progress In Electromagnetics Research, Vol. 98, 425-443, 2009.

[46] P. White, "Group delay explanations and applications”, Applied Radio Labs, pp. 1-6, 1999.
[47] Z. Abate, WiMAX RF systems engineering, Mobile communication series, Artech House 2009, Norwood, MA, USA.

[48] S.-S. Myoung, B.-S. Kwon, Y.-H. Kim and J.-G. Yook, "Effect of group delay in RF BPF on impulse radio systems", IEICE Tran. Communications, Vol. 90, No. 12, pp. 3514-3522, 2007. 